\title{
Large-Scale Direct SLAM for Omnidirectional Cameras
}

\author{
David Caruso $^{1}$ and Jakob Engel ${ }^{2}$ and Daniel Cremers ${ }^{2}$
}

\begin{abstract}
We propose a real-time, direct monocular SLAM method for omnidirectional or wide field-of-view fisheye cameras. Both tracking (direct image alignment) and mapping (pixel-wise distance filtering) are directly formulated for the unified omnidirectional model, which can model central imaging devices with a field of view above $180^{\circ}$. This is in contrast to existing direct mono-SLAM approaches like DTAM or LSDSLAM, which operate on rectified images, in practice limiting the field of view to around $130^{\circ}$ diagonally. Not only does this allows to observe - and reconstruct - a larger portion of the surrounding environment, but it also makes the system more robust to degenerate (rotation-only) movement. The two main contribution are (1) the formulation of direct image alignment for the unified omnidirectional model, and (2) a fast yet accurate approach to incremental stereo directly on distorted images. We evaluated our framework on real-world sequences taken with a $185^{\circ}$ fisheye lens, and compare it to a rectified and a piecewise rectified approach.
\end{abstract}

\section{INTRODUCTION}

Visual Odometry (VO) and Simultaneous Localization and Mapping (SLAM) are becoming increasingly important for robotics and mobile vision applications, as they only require optical cameras - which are cheap, light and versatile, and hence can easily be put into commodity hardware. A lot of research has been focused around these topics throughout the last decade, with a particular focus on real-time systems which can be used for autonomous control for example of UAVs [1], [2].

Most existing approaches are based on keypoints: Once keypoints are extracted, the images are abstracted to a collection of point-observations, which are then used to compute geometrical information. This can be done in a filtering framework [3], [4], [5], or in a keyframe-based non-linear optimization framework [6], [7], [8]. This has the advantage that a large part of the required workload only is done once on keypoint extraction, such that remaining computational resources can be spent on enforcing geometric consistency, and outliers can be removed in a straight-forward way.

More recently, so-called direct approaches have gained in popularity: instead of abstracting the images to pointobservations, the idea is to compute dense [9], or semidense [10] depth maps in an incremental fashion, and track the camera using direct image alignment. This has the advantage that much more information can be used, in particular information contained in edges or densely textured

\footnotetext{
*The research leading to these results was supported by the BMBF within the Software Campus (NanoVis) No. 01IS12057

${ }^{1}$ David Caruso is with the Ecole polytechnique, Palaiseau, France david.caruso@polytechnique.edu

${ }^{2}$ Jakob Engel and Daniel Cremers are with the Technical University Munich engelj@in.tum.de, cremers@tum.de
}

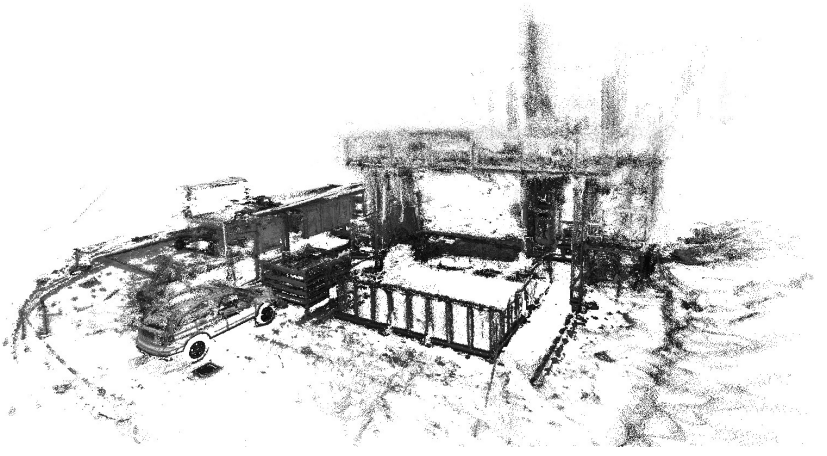

Fig. 1. Top: 3D reconstruction obtained in real-time with our approach, using a $185^{\circ}$ fisheye lens. Bottom: Map of the trajectory and set of example keyframes, with associated color-coded inverse distance maps.
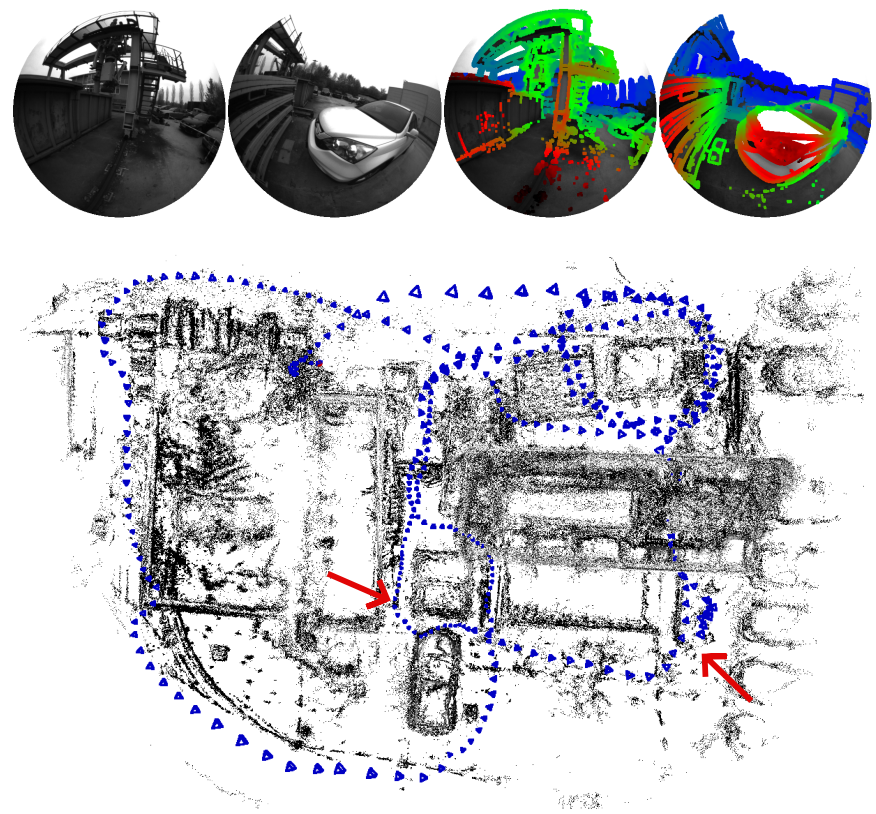

surfaces. Further, the generated map contains substantially more information about the environment, which can be used for obstacle-avoidance and path-planning.

What all these methods have in common is that they rely on a sufficiently informative environment. In many practical cases however, this can be a very restrictive assumption: For example indoors where there are many untextured white walls, or in the presence of moving objects, large parts of the camera image can become uninformative for SLAM. This is especially true if the used camera only has a small field of view (FoV). On the other hand, the wider the field of view, the more likely that some part of the visible scene is wellsuited for SLAM.

Nevertheless, most visual SLAM or VO systems are restrained to using a classical pinhole camera model. Often, 
this is combined with a radial distortion model (such as the ATAN model used in PTAM). All these models can not directly be used for omnidirectional cameras (with FoV of more than $180^{\circ}$ ). This is especially true for direct methods, which typically operate on rectified images - limiting the field of view to no more than $130^{\circ}$.

In this paper, we propose an extension of LSD-SLAM [10] to a generic omnidirectional camera model. The resulting method is capable of handling all types of central projection systems such as fisheye and catadioptric cameras. We evaluate it on images captured with a fisheye lens covering a FoV of $185^{\circ}$. We show that especially for trajectories which contain aggressive camera rotations, it outperforms methods based on a pinhole-projection model.

\section{A. Related Work}

There is a range of related work regarding omnidirectional vision, in particular for robot and ground-vehicle localization. For instance [11] uses a catadioptric system to estimates the ego-motion of a vehicle, using direct photometric error minimization for rotation estimation - it is however restricted to planar motion. In [12], RANSAC point association for SIFT features is used for estimating translation and rotation, on a rig of 5 rectified cameras. Again, the system is restricted to planar motion. In [13] a multicamera rig is used to build a topological map based on appearance. In [14] an EKFbased SLAM system is adapted for omnidirectional cameras. In [15], the advantage of using omnidirectional cameras in this context is shown. The work of Meilland et. al. [16] is somewhat closer to ours, as it performs dense registration against multiple frames from a database of spherical images. They are augmented with distance information from an external sensor or stereo-vision. However, the system is based on a priori learned database of georeferenced images and does not perform online SLAM.

\section{B. Contribution and Outline}

In this paper we explore the use of omnidirectional and fisheye cameras for direct, large-scale visual SLAM. We propose two different camera model choices, which we integrate into the recently appeared LSD-SLAM [10] framework, and evaluate the resulting algorithm on real-world and simulated data. More precisely, the main contribution of this paper is two-fold: (1) We give a direct image alignment formulation operating on an omnidirectional camera model. (2) We derive an efficient and accurate approach to perform stereo directly on omnidirectional images, both for the piecewise rectification approach and natively on the Unified Omnidirectional Model. We intend to make the used datasets including ground-truth publicly available.

The paper is organized as follows: In Chapter II we introduce a camera model as general projection function, and describe the three parametrized models considered in this paper: The Pinhole Model, an Array of Pinhole Model, and the Unified Omnidirectional Model. In Chapter III, we describe our omnidirectional direct SLAM method. We start by reviewing the LSD-SLAM pipe-line as introduced in [10].

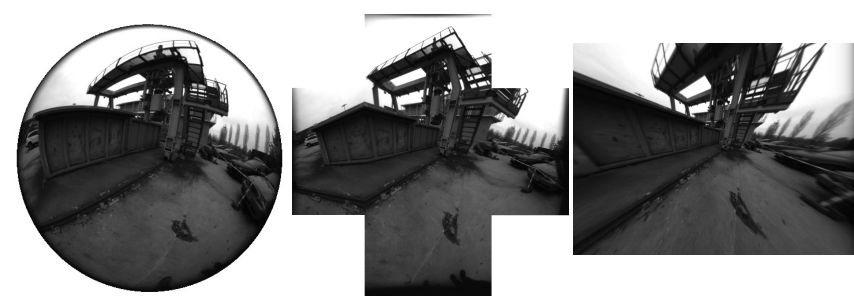

Fig. 2. Camera Models : The same image, warped to fit the three projection models considered in this paper. Both the Unified model and the piecewise rectified model can cover the full $185^{\circ}$ field-of-view. For the pinhole model, the image was cropped to a horizontal field of view of $120^{\circ}$, which still causes significant stretching of the image around the border.

We then detail how the two major steps that depend on the camera model - probabilistic, semi-dense depth estimation and direct image alignment - are adapted to operate in realtime on images from omni-directional cameras. In Chapter IV] we evaluate the accuracy, robustness and runtime for the three different models on a both simulated and real-world data. Finally, in Chapter $\mathrm{V}$, we summarize the results and line out future work.

\section{CAMERA MODELS}

In this chapter, we will lay out the three different parametric projection functions $\pi$ considered in the paper: In Sec. II-A, we briefly review the well-known Pinhole Model and discuss its limitations. We then extend it to a more general Array of Pinhole Model allowing to cover the full viewing sphere in Sec. II-B. In Sec. II-C, we introduce the Unified Omnidirectional Model, which allows to model 360-vision in closed-form.

Notation. We use bold, capital letters $\boldsymbol{R}$ to denote matrices, and bold, lower-case letters $\mathbf{x}$ for vectors. $\mathbf{u}=[u, v]^{T} \in \Omega \subset \mathbb{R}^{2}$ will generally denote pixel coordinates, where $\Omega$ denotes the image domain. $\mathbf{x}=[x, y, z]^{T} \in \mathbb{R}^{3}$ will be used for $3 \mathrm{D}$ point coordinates.

In the most general case, a camera model is a function $\pi: \mathbb{R}^{3} \rightarrow \Omega$, which defines the mapping between $3 \mathrm{D}$ points $\mathbf{x}$ in the camera frame, and pixels $\mathbf{u}$ in the image. For lenses with negligible diameter, a common assumption is the single viewpoint assumption, i.e., that all light-rays pass through a single point in space - the origin of the camera frame $\mathcal{C}$. Hence, the projected position of the point only depends on the direction of $\mathbf{x}$. We will use $\pi^{-1}: \Omega \times \mathbb{R}^{+} \rightarrow \mathbb{R}^{3}$ for the function mapping pixels back to $3 \mathrm{D}$, using their inverse distance $d=\|\mathbf{x}\|^{-1}$.

Note that the single viewpoint assumption allows transforming images from any camera model to any other, for the domain of visible points they have in common - this is generally referred to as image rectification, and is a frequently done preprocessing step, transforming the image to follow a more simple model e.g. by removing radial distortion. Given two projection functions $\pi_{1}, \pi_{2}$ and an image $I_{1}: \Omega_{1} \rightarrow \mathbb{R}$ taken with a camera $\pi_{1}$, we can compute the respective image $I_{2}: \Omega_{2} \rightarrow \mathbb{R}$ following projection $\pi_{2}$ as

$$
I_{2}(u, v)=I_{1}\left(\pi_{1}\left(\pi_{2}^{-1}(u, v, 1)\right)\right) .
$$




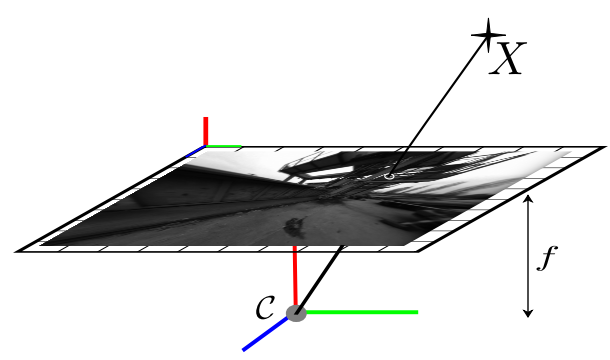

Fig. 3. Pinhole Model. A 3D point is directly projected onto the image plane through $\mathcal{C}$.

This warping however introduces interpolation artifacts and can degrade the image quality, especially in areas where the angular resolution changes significantly.

\section{A. Pinhole Model}

The pinhole camera model is the most used camera model. The image is obtained by projecting each point onto a plane located at $z=1$, followed by an affine mapping

$$
\pi_{p}(\mathbf{x}):=\left[\begin{array}{cc}
f_{x} & 0 \\
0 & f_{y}
\end{array}\right]\left[\begin{array}{l}
x / z \\
y / z
\end{array}\right]+\left[\begin{array}{l}
c_{x} \\
c_{y}
\end{array}\right]
$$

where $f_{x}, f_{y}$ are the focal lengths, and $c_{x}, c_{y}$ is the principal point. It is schematically shown in Fig. 3 .

This model is often used as the linearity of the projection function (in homogeneous coordinates) - and the fact that straight lines in 3D are projected to straight lines in the image - make it the most simple model choice to use. It however has the major drawback that it cannot model a wide field of view: The angular resolution increases drastically towards the borders of the image, leading to a distorted image - an example is shown on the right in Fig. 2

In order to make this model compatible to small radial distortions, a non-linear radial distortion function - often approximated polynomially - can be applied to the projected pixel coordinates.

\section{B. Array of Pinhole Camera}

A straight-forward approach to extending the field of view is to use a camera model consisting of an array of several pinhole cameras, which have the same principal point but different orientations. The projection function $\pi_{m p}(\mathbf{x}): \mathbb{R}^{3} \rightarrow$ $\cup_{i} \Omega_{i}$ is then given by piecewise rotation followed by pinhole projection, i.e.,

$$
\pi_{m p}(\mathbf{x}):=\pi_{p_{i(\mathbf{x})}}\left(\mathbf{R}_{i(\mathbf{x})} \mathbf{x}\right)
$$

where $i(\mathbf{x}): \mathbb{R}^{3} \rightarrow[1, k]$ segments the $3 \mathrm{D}$ space into $k$ subspaces. While in general the segmentation and orientation of the associated cameras can be chosen arbitrarily, we choose to split $\mathbb{R}^{3}$ into six equally sized quadrants, forming a cubeshaped image plane as seen on Fig. 4 This has the advantage that $i(\mathbf{x})$ can be computed from binary comparisons on $x, y$ and $z$, while the $\mathbf{R}_{i}$ correspond to orthogonal rotations.

While this model has a number of desirable properties it is piecewise linear in homogeneous coordinates, simple to compute and offers reasonably homogeneous angular resolution - it does not fit natural lenses. In order to use

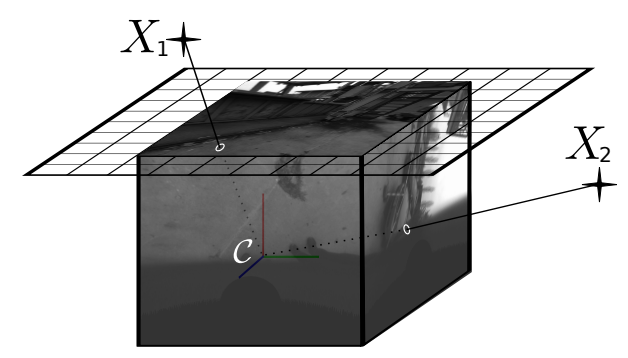

Fig. 4. Piecewise Pinhole Model. A 3D point is projected through the center of the camera on one of the image planes depending on the subspace it lies in, effectively forming a cube-shaped image plane. $X_{1}$ and $X_{2}$ are projected to different images $\Omega_{1}$ and $\Omega_{2}$.

it, incoming images have to be rectified in a preprocessing step. Further, the piecewise nature of the model causes discontinuities in the image space $\Omega=\cup_{i} \Omega_{i}$, complicating its use in practice.

\section{Central Omnidirectionnal Camera: Unified Model}

A number of different projection functions has been proposed in the literature for modeling and calibrating catadioptric and dioptric omnidirectional cameras. Desirable properties of such a function include (1) its capability to accurately describe a wide range of actual physical imaging devices, (2) the ease of parameter calibration and (3) the existence of a closed-form expression for the unprojection function $\pi^{-1}$. As this paper targets real-time direct SLAM, an additional criterion is the computational cost of projecting and unprojecting points, as well as the cost of evaluating the corresponding derivatives.

Accurate results were obtained by moving all nonlinearities into a radially symmetric function, and identifying the first coefficients of its Taylor expansion [17]. While this approach can model every camera that fits the single viewpoint assumption, it lacks a closed-form unprojection function - and approximating it is computationally costly.

Instead, we use the model originally proposed in [18] for central catadioptric systems and extended in [19], [20] for a wider range of physical devices including fisheye cameras. The central idea behind this model is to concatenate two successive projections as depicted on Fig. 5. The first one projects the point from the world onto a camera-centered unit sphere. The second one is an ordinary pinhole projection trough a center shifted along the $\mathrm{z}$ axis by $-\xi$. This model is described by a total of five parameters, $f_{x}, f_{y}, c_{x}, c_{y}$ and $\xi$. The projection of a point is computed as

$$
\pi_{u}(\mathbf{x})=\left[\begin{array}{c}
f_{x} \frac{x}{z+\|\mathbf{x}\| \xi} \\
f_{y} \frac{y}{z+\|\mathbf{x}\| \xi}
\end{array}\right]+\left[\begin{array}{c}
c_{x} \\
c_{y}
\end{array}\right],
$$

where $\|\mathbf{x}\|$ is the euclidean norm of $\mathbf{x}$. The corresponding unprojection function can be computed in closed form, and is given by

$$
\pi_{u}^{-1}(\mathbf{u}, d)=\frac{1}{d}\left(\frac{\xi+\sqrt{1+\left(1-\xi^{2}\right)\left(\hat{u}^{2}+\hat{v}^{2}\right)}}{\hat{u}^{2}+\hat{v}^{2}+1}\left[\begin{array}{c}
\hat{u} \\
\hat{v} \\
1
\end{array}\right]-\left[\begin{array}{l}
0 \\
0 \\
\xi
\end{array}\right]\right)
$$




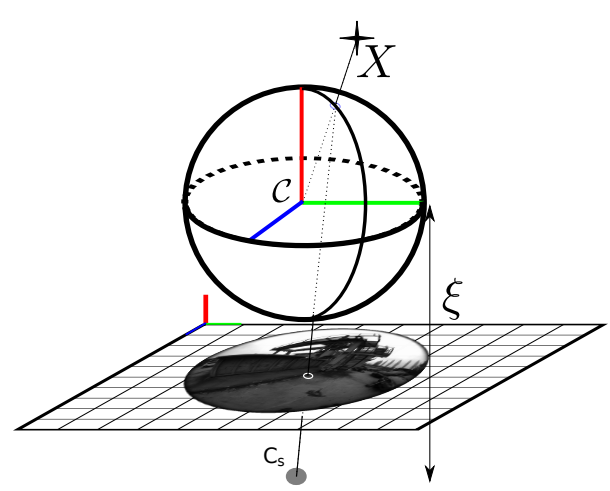

Fig. 5. Unified Model. A 3D point is first projected on the unit sphere, and then the image plane via a secondary, shifted camera center $C_{S}$.

where

$$
\left[\begin{array}{l}
\hat{u} \\
\hat{v}
\end{array}\right]=\left[\begin{array}{l}
\left(u-c_{x}\right) / f_{x} \\
\left(v-c_{y}\right) / f_{y}
\end{array}\right] .
$$

One major advantage of this model is the availability of an easy-to-compute projection and unprojection function and its derivatives. Note that for $\xi=0$ it reduces to the pinhole model. In order to improve the generality of the model, we combine it with a small radial-tangential distortion to correct lens imperfections - similar to the pinhole case, images are warped once in the beginning, to perfectly fit this model.

\section{DIRECT OMNIDIRECTIONAL SLAM}

In this Chapter, we describe our omnidirectional, largescale direct SLAM system, which is based on LSD-SLAM [10]. First, in Sec. III-A we review the LSD-SLAM pipeline adapted to omnidirectional cameras. We extend the direct image registration of LSD-SLAM to omnidirectionnal camera in Sec. III-B In Sec. III-D we show how - in this framework - stereo can be done efficiently on the unified (1) and piecewise rectified (2) model.

Notation. $\quad D: \Omega_{d} \rightarrow \mathbb{R}^{+}$will denote the inverse distance map of the current keyframe.With a slight abuse of notation, elements of $\mathfrak{s e}(3) / \mathfrak{s i m}(3)$ will directly be represented as vector $\boldsymbol{\mu}$, and we use exp and log to associate an element of the lie algebra to the corresponding element of the lie group. We then define the composition operator $\circ$ as

$$
\boldsymbol{\mu}_{\mathbf{1}} \circ \boldsymbol{\mu}_{\mathbf{2}}:=\log \left(\exp \left(\boldsymbol{\mu}_{1}\right) \cdot \exp \left(\boldsymbol{\mu}_{2}\right)\right) \text {. }
$$

As a shorthand, we use $\mathbf{R}_{\boldsymbol{\mu}}$ and $\mathbf{t}_{\boldsymbol{\mu}}$ to denote the corresponding rotation matrix and translation vector of a transformation.

\section{A. Method Overview}

Our method continuously builds and maintains a posegraph of keyframes. Each keyframe contains a probabilistic semi-dense inverse distance map, which maintains a Gaussian probability distribution over the inverse distance for all pixels which have sufficient intensity gradient. It is estimated over time by filtering over a large number of small-baseline stereo comparisons. In turn, new camera poses - as well as loop-closure constraints - are computed using direct image alignment. Note that in contrast to [10], we use the inverse distance $d=\|\mathbf{x}\|^{-1}$ instead of depth, such that we can model points behind the camera. An overview is shown in Fig. 6

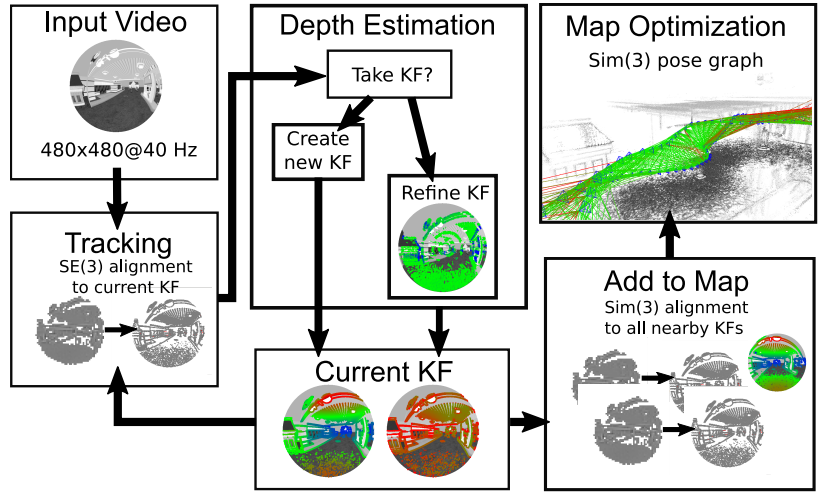

Fig. 6. Overview over the LSD-SLAM pipeline for omnidirectional Cameras. Tracking and depth estimation inherently depend on the camera model used, their omnidirectional versions and are detailed in Sec. III-B and III-D receptively.

1) $S E$ (3) Tracking: When a new camera frame is captured, its rigid-body pose relative to the closest keyframe is tracked using direct image alignment, which will be described in Sec. III-B

2) Probabilistic Distance Map Estimation: Keyframes are selected at regular intervals, based on the moved distance to the previous keyframe (relative to its mean inverse distance), as well as the relative overlap. For each keyframe, an inverse distance map is initialized by propagating the inverse distance map from its immediate predecessor. Subsequently, it is updated - and extended to new regions - by incorporating information obtained from many small-baseline stereo comparisons. This step will be described in more detail in Sec. III-D

3) Scale-Drift Aware Pose-Graph Optimization: In the background, we continuously perform pose graph optimization between all keyframes, and attempt to find new constraints between keyframes which are likely to overlap. Constraints are expressed as similarity transforms to account for scale-drift - for more details see [10].

4) Initialization: The system is initialized with a random depth map with mean one and a large covariance - this generally converges to a good estimate, as long as the camera motion within the first few seconds is not degenerate.

\section{B. Omnidirectional Direct Image Alignment on SE(3)}

Every new frame $I_{\text {new }}$ is first tracked relative to the closest keyframe $I_{\mathrm{Kf}}$ with associated inverse distance map $D_{\mathrm{Kf}}$ by direct minimization of the photometric error, defined as

$$
E^{\text {frame }}(\boldsymbol{\mu}):=\sum_{\mathbf{u} \in \boldsymbol{\Omega}_{\mathbf{d}}} \rho\left(\frac{r_{\mathbf{u}}^{I}(\boldsymbol{\mu})}{\sigma_{r_{\mathbf{u}}^{I}(\boldsymbol{\mu})}}\right),
$$

where $\rho$ denotes the robust Huber norm, and

$$
\begin{aligned}
r_{\mathbf{u}}^{I}(\boldsymbol{\mu}) & =I_{\mathrm{Kf}}(\mathbf{u})-I_{\text {new }}(\pi(\omega(\boldsymbol{\mu}, \mathbf{u}))) \\
\omega(\boldsymbol{\mu}, \mathbf{u}) & =\mathbf{R}_{\boldsymbol{\mu}} \pi^{-1}\left(\mathbf{u}, D_{\mathrm{Kf}}(\mathbf{u})\right)+\mathbf{t}_{\boldsymbol{\mu}} .
\end{aligned}
$$

The function $\omega$ unprojects a point, and transforms it by $\boldsymbol{\mu}$. As in [10], the residuals are normalized with their propagated inverse distance variance. 
This weighted least-squares problem is then minimized in a coarse-to-fine scheme using the iteratively re-weighted Levenberg-Marquardt algorithm in a left-compositional formulation: In each iteration, we solve for a left-multiplied increment

$$
\boldsymbol{\delta} \boldsymbol{\mu}^{(k)}=\left(\mathbf{J}^{T} \mathbf{W} \mathbf{J}+\lambda \operatorname{diag}\left(\mathbf{J}^{T} \mathbf{W} \mathbf{J}\right)\right)^{-1} \mathbf{J}^{T} \mathbf{W r},
$$

where $\mathbf{r}=\left[\mathbf{r}_{\mathbf{u}_{1}}^{I} \ldots \mathbf{r}_{\mathbf{u}_{n}}^{I}\right]^{T}$ is the stacked residual vector and $\mathbf{W}$ a diagonal matrix containing the weights. $\mathbf{J}$ is the $n \times 6$ Jacobian of the stacked residual vector evaluated at $\boldsymbol{\mu}^{(k)}$ :

$$
\mathbf{J}=\frac{\partial \mathbf{r}\left(\boldsymbol{\epsilon} \circ \boldsymbol{\mu}^{(k)}\right)}{\partial \boldsymbol{\epsilon}}
$$

which is then left-multiplied on the current estimate

$$
\boldsymbol{\mu}^{(k+1)}=\boldsymbol{\delta} \boldsymbol{\mu}^{(k)} \circ \boldsymbol{\mu}^{(k)} .
$$

Using the chain rule, each $1 \times 6$ row $\mathbf{J}_{\mathbf{u}}$ of the Jacobian can be decomposed into three parts

$$
\mathbf{J}_{\mathbf{u}}^{\mathrm{fwd}}=-\left.\left.\left.\mathbf{J}_{I_{\text {new }}}\right|_{\pi} \mathbf{J}_{\pi}\right|_{\omega} \mathbf{J}_{\omega}\right|_{\mu},
$$

where

- $\left.\mathbf{J}_{\omega}\right|_{\boldsymbol{\mu}^{(k)}}$ is a $3 \times 6$ Jacobian, denoting the leftcompositional derivative of the transformed point, evaluated at $\boldsymbol{\mu}=\boldsymbol{\mu}^{(k)}$

$$
\left.\mathbf{J}_{\omega}\right|_{\mu}=\frac{\partial \omega(\boldsymbol{\epsilon} \circ \boldsymbol{\mu}, \mathbf{u})}{\partial \boldsymbol{\epsilon}} .
$$

- $\left.\mathbf{J}_{\pi}\right|_{\omega}$ is the $2 \times 3$ Jacobian of the projection function $\pi$ evaluated at $\omega=\omega\left(\boldsymbol{\mu}^{(k)}, \mathbf{u}\right)$.

- $\left.\mathbf{J}_{I_{\text {new }}}\right|_{\pi}$ is the $1 \times 2$ intensity gradient of the new image, evaluated at point $\pi=\pi\left(\omega\left(\boldsymbol{\mu}^{(k)}, \mathbf{u}\right)\right)$.

Notice how the evaluation point of each of these Jacobians depends on $\boldsymbol{\mu}^{(k)}$, hence everything has to be re-evaluated in each iteration. In practice, the computational cost is the dominated by this evaluation - which is especially true in our case, as for the unified model the projection, and hence its derivative $\left.\mathbf{J}_{\pi}\right|_{\omega}$ is much more complex.

To avoid this, we use an inverse compositional formulation - a trick that is well known in the literature [21]: In each iteration, instead of applying the increment to the points in the reference frame, its inverse is applied to the points in the keyframe. That is, instead of linearizing

$$
I_{\mathrm{Kf}}(\mathbf{u})-I_{\text {new }}\left(\pi\left(\omega\left(\boldsymbol{\epsilon} \circ \boldsymbol{\mu}^{(k)}, \mathbf{u}\right)\right)\right),
$$

with respect to $\epsilon$, we linearize

$$
I_{\mathrm{Kf}}(\pi(\omega(\boldsymbol{\epsilon}, \mathbf{u})))-I_{\text {new }}\left(\pi\left(\omega\left(\boldsymbol{\mu}^{(k)}, \mathbf{u}\right)\right)\right) .
$$

The Jacobian now becomes

$$
\mathbf{J}_{\mathbf{u}}^{\mathrm{bkwd}}=\left.\left.\left.\mathbf{J}_{I_{\mathrm{Kf}}}\right|_{\pi} \mathbf{J}_{\pi}\right|_{\omega} \mathbf{J}_{\omega}\right|_{\mathbf{0}},
$$

with $\omega=\omega(\mathbf{0}, \mathbf{u})$ and $\pi=\pi(\omega(\mathbf{0}, \mathbf{u}))$. It is thus independent of $\boldsymbol{\mu}^{(k)}$. This allows us to precompute it once per pyramid level, saving much of the required computations. Note that we still have to re-evaluate the outer product $\mathbf{J}^{T} \mathbf{W J}$ on each iteration, as the weight matrix changes. The inverse of the resulting update is then right-multiplied onto the current estimate, i.e.,

$$
\boldsymbol{\mu}^{(k+1)}=\boldsymbol{\mu}^{(k)} \circ\left(-\boldsymbol{\delta} \boldsymbol{\mu}^{(k)}\right) .
$$

Note that for the forward compositional as well as the inverse compositional formulation, both projection and unprojection appear in the error function - motivating the choice of a camera model for which both can be expressed in closedform. This is in contrast to classical Bundle Adjustment, where only the projection function - but not its inverse appears in the error function.

\section{Omnidirectional Direct Image Alignment on Sim(3)}

In monocular SLAM, the absolute scale is not observable and drifts over time - which has to be taken into account when finding loop-closures. As in [10], we use $\operatorname{Sim}(3)$ image alignment between keyframes, to estimate not only their relative pose, but also the scale difference between their inverse distance maps. This is done by introducing an additional error term - the geometric error - which penalizes differences in inverse distance. The energy function for aligning $\left(I_{\mathrm{K} 1}, D_{\mathrm{K} 1}\right)$ and $\left(I_{\mathrm{K} 2}, D_{\mathrm{K} 2}\right)$ thus becomes

$$
E^{\mathrm{Kf}}(\boldsymbol{\mu}):=\sum_{\mathbf{u} \in \boldsymbol{\Omega}_{\mathbf{d}}}\left[\rho\left(\frac{r_{\mathbf{u}}^{I}(\boldsymbol{\mu})}{\sigma_{r_{\mathbf{u}}^{I}(\boldsymbol{\mu})}}\right)+\rho\left(\frac{r_{\mathbf{u}}^{D}(\boldsymbol{\mu})}{\sigma_{r_{\mathbf{u}}^{D}(\boldsymbol{\mu})}}\right)\right],
$$

where $\boldsymbol{\mu} \in \mathfrak{s i m}(3)$, and

$$
\begin{aligned}
r_{\mathbf{u}}^{I}(\boldsymbol{\mu}) & =I_{\mathrm{K} 1}(\mathbf{u})-I_{\mathrm{K} 2}\left(\pi\left(\omega_{s}(\boldsymbol{\mu}, \mathbf{u})\right)\right) \\
r_{\mathbf{u}}^{D}(\boldsymbol{\mu}) & =\left\|\omega_{s}(\boldsymbol{\mu}, \mathbf{u})\right\|^{-1}-D_{\mathrm{K} 2}\left(\pi\left(\omega_{s}(\boldsymbol{\mu} . \mathbf{u})\right)\right) .
\end{aligned}
$$

Note that we now optimize over relative scale as well, and hence have to apply a similarity warp, defined as

$$
\omega_{s}(\boldsymbol{\mu}, \mathbf{u})=s_{\boldsymbol{\mu}} \mathbf{R}_{\boldsymbol{\mu}} \pi^{-1}\left(\mathbf{u}, D_{\mathrm{K} 1}(\mathbf{u})\right)+\mathbf{t}_{\boldsymbol{\mu}},
$$

where $s_{\boldsymbol{\mu}}$ is the scaling factor of $\boldsymbol{\mu}$. Note that in contrast to [10], this residual now penalizes differences in inverse distance. Again, we apply statistical normalization based on the propagated variances as in [10]. For tracking $\operatorname{Sim}(3)$ constraints, we use a forward-compositional formulation.As in [10], the approximated Hessian $\left(\mathbf{J}^{T} \mathbf{W J}\right)^{-1}$ of the last iteration can be interpreted as covariance on a left-multiplied increment on $\boldsymbol{\mu}$, and is used in the subsequent pose-graph optimization.

\section{Semi-Dense Depth Map Estimation}

Once a frame is registered to a keyframe, stereo matching is performed to refine the keyframe distance map $D_{\mathrm{Kf}}$. As matching cost we use the sum of squared differences (SSD) over five equidistant pixels along the epipolar line. If a prior exists, the epipolar search is constrained to the interval $\left[d-2 \sigma_{d}, d+2 \sigma_{d}\right]$. This greatly improves efficiency and minimizes the probability of finding an incorrect match, as in practice only very short line segments have to be searched. Subsequently, we refine the found match to subpixel precision.

Similar to [22], each new measurement is fused into the existing depth map. Measurement variances $\sigma_{m}^{2}$ are obtained using the geometric and photometric error, as derived in [22]. 


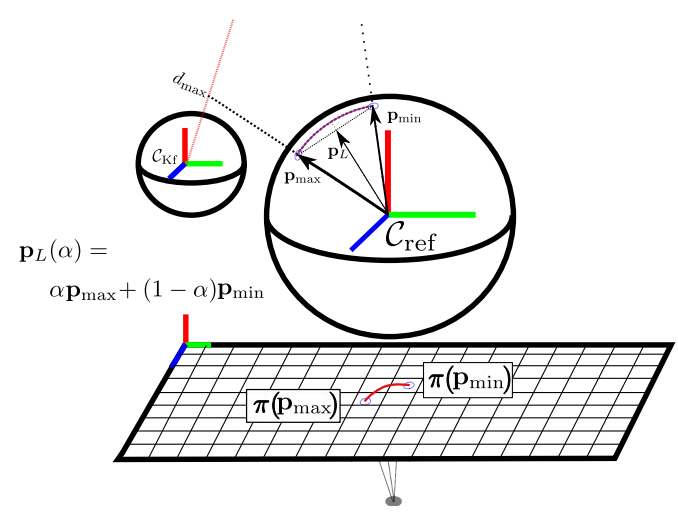

Fig. 7. Non-Rectified Stereo Matching: We efficiently browse the epipolar curve in the image $\mathbf{u}_{L}$ using a parametric equation. It is obtained by projecting the line connecting $\mathbf{p}_{\max }$ and $\mathbf{p}_{\min }$ on the unit sphere around the camera center.

Finally, we smooth the inverse distance map, and remove outliers.

1) Non-Rectified Stereo: When performing stereo on the unified model, epipolar lines are not straight lines, but curves. More precisely, Geyer et al. showed that these epipolar curves are conics [18], as they are the pinhole-projection of a geodesic on the unit sphere, as visualized in Fig. 77. We here present a general method to incrementally and efficiently compute points along the epipolar curve, at a constant stepsize of $1 \mathrm{px}$ : While this is trivial for straight lines, it is not straight-forward for the general case of epipolar curves.

We first define the two points $\mathbf{p}_{\max }, \mathbf{p}_{\min } \in \mathbb{R}^{3}$ on the unit sphere around the projective center $\mathcal{C}_{\text {ref }}$, which correspond to the maximum and minimum inverse distance of the search interval $d_{\max }, d_{\min }$ :

$$
\begin{aligned}
\mathbf{p}_{\max } & :=\pi_{s}\left(\mathbf{R} \pi_{u}^{-1}\left(\mathbf{u}, d_{\max }\right)+\mathbf{t}\right) \\
\mathbf{p}_{\min } & :=\pi_{s}\left(\mathbf{R} \pi_{u}^{-1}\left(\mathbf{u}, d_{\min }\right)+\mathbf{t}\right) .
\end{aligned}
$$

Here, $\pi_{s}$ projects a point onto the unit sphere, $\pi_{u}^{-1}$ is the unprojection function of the unified model (5), and $\mathbf{u}$ is the pixel in $I_{\mathrm{Kf}}$ we are trying to match. We then express the straight line between these points as

$$
\mathbf{p}_{L}(\alpha)=\alpha \mathbf{p}_{\max }+(1-\alpha) \mathbf{p}_{\min },
$$

for $\alpha \in[0,1]$. This also gives a parametric expression for the epipolar curve in $I_{\text {ref }}$ as

$$
\mathbf{u}_{L}(\alpha):=\pi_{u}\left(\mathbf{p}_{L}(\alpha)\right) .
$$

Note that we apply the full unified projection function, which first projects a point $\mathbf{p}_{L}$ onto the geodesic, and then into the image. This is visualized in Fig. 7 .

Starting at $\mathbf{u}_{L}(0)$, we then browse the epipolar curve by incrementing $\alpha$. A step-size of 1 pixel is enforced by using a first-order Taylor expansion of $\mathbf{u}_{L}$, and choosing the increment in $\alpha$ as

$$
\delta \alpha=\left\|\left.\mathbf{J}_{\mathbf{u}_{L}}\right|_{\alpha}\right\|^{-1},
$$

which we re-evaluate for each increment. Note that this method is independent of the shape of the epipolar curve, and hence can be used for any central camera model.

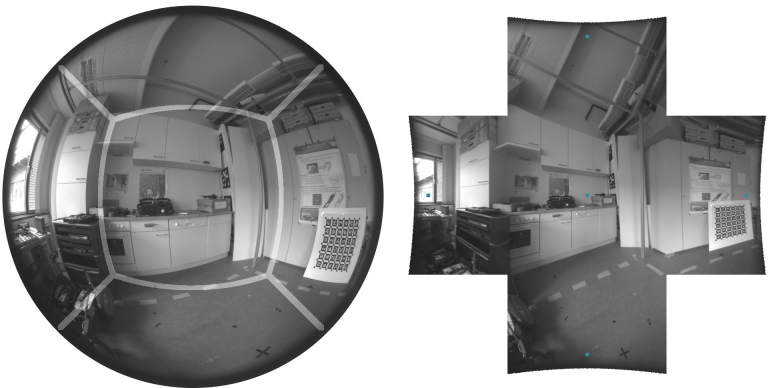

Fig. 8. Piecewise rectification: Example of fisheye camera rectification The borders are still significantly distorted compared to the original image, as it is clearly visible on the checker-board, which leads to interpolation artifacts or blur.

Nevertheless, it is much more expensive than browsing a straight line, as each point is projected individually. In LSDSLAM however, the search interval is always small, as either a good prior is available, or the pixel has just been initialized and hence the baseline is small.

2) Pre-Rectified Stereo: For a large disparity search range, the above method can become very costly since it requires re-evaluation of the projection function for each point. Thus, the valid question arises whether piecewise rectification of the input image as described in Sec II-B, followed by straight-forward line-browsing would be faster. For this we determined suitable values for the focal lengths $f_{x}$ and $f_{y}$ of each pinhole camera individually, minimizing the change in angular resolution at each point in the image. An example is shown in Fig. 8. Still, some distortion is clearly visible, note for example how the checker-board shape is altered. Further, we extend the visible field of each rectified image by 20 pixels images, which is not displayed in the figure. We then perform line-stereo the same way as is done in [22]. In Sec. IV we will compare these two approaches regarding accuracy and efficiency.

\section{RESULTS}

We evaluate our algorithm regarding accuracy and computational requirements on both synthetic and real data.

\section{A. Hardware Setup}

For real data experiment, we use a global-shutter usb3 camera equipped with a $185^{\circ}$ FoV fisheye lens. The $\xi$ parameter for this system has been estimated to 2.06 by offline calibration, using the Kalibr toolbox [23]. Images are cropped and scaled to a $480 \times 480$ region centered around the principal point. We recorded a number of trajectories with rapid, handheld motion, including quick rotation - Figure 9 shows some example images from the longest sequence. We also show two of the sequences (T2 and T5) in the attached video. For ground truth acquisition, we use a motion capture system which covers an area of approximately $7 \times 12 \mathrm{~m}$ - as some trajectories leave this area, we only compute errors on the part for which ground truth data is available. The synthetic data was generated using Gazebo simulator, modified to have as extra output the synchronized pose, $185^{\circ}$ images, and distance ground truth. The movement is slower on this dataset and mimics that of a quadrotor. 


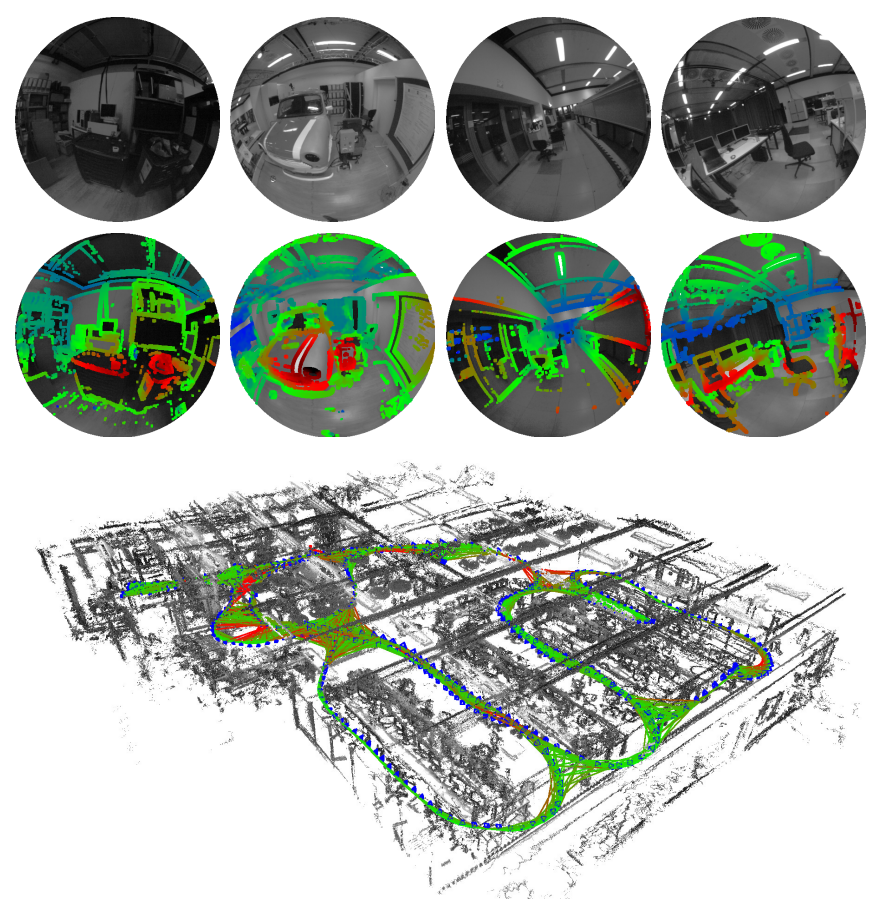

Fig. 9. Reconstruction of 75 sequence. Top: Color-coded inverse distance maps. Note how we can obtain geometry for the full $185^{\circ}$ field of view. Bottom: Final point cloud. This corresponds to the right plot in Fig. 10

For comparison with a pinhole model, we also synthesize a sequence of rectified images, artificially cropping the field of view to $100^{\circ}$ horizontally and vertically. We make the dataset including ground truth publicly available online ${ }^{1}$

\section{B. Evaluated Parameters}

We evaluate the effect of three different parameters:

- Camera Model: We use either the unified omnidirectional model (Uni, Sec. III-D.1), or a piecewise rectified model (Multi, Sec. III-D.2). As baseline, we use the cropped \& rectified video with a pinhole model (Pin).

- Input Resolution: We use either an input resolution of $480 \times 480$ (Full) or $240 \times 240$ (Half).

- Resolution Used for Tracking: We choose to stop the coarse to fine approach in tracking either at the input image (level 0 of the image pyramid) or at the first octave of it (level 1), allowing to speed-up tracking significantly, while maintaining most of the accuracy.

For comparison we also run the recently presented ORBSLAM [24] on the rectified dataset $(O r b)$. All the experiments were conducted on a Intel i7 laptop CPU.

\section{Accuracy Comparison}

We evaluate the accuracy of our method in terms of the translational root mean square error (RMSE) of the final position of all keyframes, after 7DoF alignment with the ground-truth. Due to the hard real-time constraint and the multi-threaded nature of the evaluated algorithms, the results are non-deterministic. In fact, small changes may cause different frames to be selected as keyframes, which in turn can greatly affect the outcome of the overall algorithm - in

${ }^{1}$ https://vision.in.tum.de/omni-lsdslam
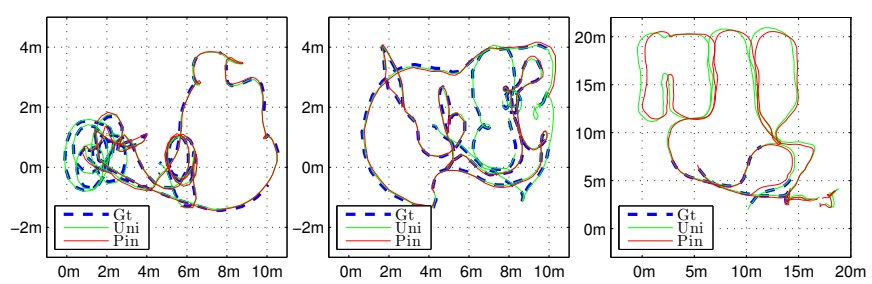

Fig. 10. Horizontal position for T2, T3 and T5. The red line shows the result of Uni-Full-0, the green line that of Pin-Full-0, and the blue dotted line the ground truth where available. For $T 2$ and $T 3$, the pinhole version is lost for a large portion of the trajectory, as they include fast rotations which cannot be tracked well with the cropped field of view. See Tab. II and Fig. 9 for an example pointcloud.

TABLE I

MEAN TIMING RESULTS (MS PER FRAME)

\begin{tabular}{lccc|ccc|cc|cc} 
& \multicolumn{3}{c|}{$480 \times 480$} & \multicolumn{3}{c|}{$240 \times 240$} & \multicolumn{3}{c}{$160 \times 160$} \\
\cline { 2 - 9 } & Mul & Uni & Pin & Mul & Uni & Pin & Mul & Uni & Pin \\
\hline Mapping & 31 & 28 & 20 & 11 & 8 & 7 & - & - & - \\
Tracking & 24 & 24 & 17 & 10 & 10 & 6 & 3 & 3 & 2.2 \\
\hline
\end{tabular}

particular for sequences containing rapid motion and strong camera rotation, both ORB-SLAM as well as LSD-SLAM behave very non-deterministic, and occasionally fail entirely. We therefore average the RMSE of the best 3 runs out of 5, in order to reduce the impact of occasional total tracking failure. The results are shown in Tab. II and some representative plots are shown in Fig. 10. Also see the attached video. If an algorithm is in a lost state for more than half of the sequence, we mark this run as failed. If more than two out of the 5 runs fail, we report it in the table.

Two things can be observed: First, results obtained with the omnidirectional camera clearly outperform the pinhole model. This shows that our algorithm can benefit from additional information in the image due to an increased field of view - in some cases very significant difference is not surprising: a wider field a view increases greatly the duration during which 3D points are visible.

The other observation is also little surprising: A higher resolution gives consistently better results than a lower resolution, although the difference is surprisingly small. Interestingly, both half resolution omnidirectional methods often outperform the full resolution pinhole model. It shows that, at least in challenging scenes, large field of view can be more important than a high image resolution, even if it leads to lower angular resolution. A more thorough evaluation of the effect of image resolution, steering the trade-off between accuracy and computational cost for direct SLAM can be found in [25]. An example of a 3D reconstruction of the synthetic scene using half and full resolution is shown in Fig. 11

\section{Timing Measurement}

Table I shows the measured average time taken by tracking and mapping, measured on the same dataset used for the accuracy evaluation. These results show that our distorted stereo matching algorithm is slightly more efficient than 
TABLE II

ABSOLUTE RMSE IN METERS

\begin{tabular}{lccccccc} 
& $\mathrm{T} 1$ & $\mathrm{~T} 2$ & $\mathrm{~T} 3$ & $\mathrm{~T} 4$ & $\mathrm{~T} 5$ & $\mathrm{~S} 1$ & $\mathrm{~S} 2$ \\
\hline Mul-Full-0 & 0.0489 & 0.0650 & $\mathbf{0 . 0 4 5 4}$ & $\mathbf{0 . 0 4 1 8}$ & 0.0501 & $\mathbf{0 . 0 2 0 1}$ & $\mathbf{0 . 0 3 9 4}$ \\
\hline Mul-Full-1 & $\mathbf{0 . 0 4 8 0}$ & 0.0693 & 0.0471 & 0.0475 & 0.0564 & 0.0365 & 0.0744 \\
\hline Mul-Half-0 & 0.0721 & 0.0963 & 0.0545 & 0.0541 & 0.0842 & 0.0206 & 0.0422 \\
\hline Mul-Half-1 & 0.0717 & 0.0967 & 0.0646 & 0.0918 & 0.1142 & 0.0319 & 0.1010 \\
\hline \hline Uni-Full-0 & 0.0524 & $\mathbf{0 . 0 5 0 0}$ & 0.0457 & 0.0448 & $\mathbf{0 . 0 3 5 5}$ & 0.0298 & 0.0418 \\
\hline Uni-Full-1 & 0.0499 & 0.0627 & 0.0490 & 0.0509 & 0.0519 & 0.0420 & 0.0681 \\
\hline Uni-Half-0 & 0.0654 & 0.0718 & 0.0563 & 0.0581 & 0.0665 & 0.0359 & 0.0491 \\
\hline Uni-Half-1 & 0.0744 & 0.0829 & 0.0588 & 0.0714 & 0.1163 & 0.0400 & 0.0635 \\
\hline \hline Pin-Full-0 & 0.0639 & 0.0799 & Failed & Failed & 0.2829 & 0.0439 & 0.6354 \\
\hline Pin-Full-1 & 0.0657 & 0.0833 & 0.0662 & 1.6509 & 0.5095 & 0.0788 & 0.5901 \\
\hline Pin-Half-0 & Failed & Failed & 0.2946 & 0.0719 & 1.2703 & 0.1157 & 2.4272 \\
\hline Pin-Half-1 & 0.8727 & Failed & 0.1089 & 0.0879 & 2.2642 & 0.1570 & Failed \\
\hline \hline Orb-Full & 0.1276 & 0.6967 & 0.4873 & 0.5238 & 0.1379 & - & - \\
\hline Orb-Half & 0.7791 & Failed & 2.3810 & 1.1385 & 0.6004 & - & - \\
\hline \hline
\end{tabular}

the multi-rectified version. This is due to the rectification required beforehand, and the fact that almost always, the browsed epipolar segments do not exceed a couple of pixels in length. Real time is easily achieved since each frame can be tracked at least $40 \mathrm{~Hz}$, and mapped at more than $30 \mathrm{~Hz}$ for a $480 \times 480$ image.

\section{CONCLUSION}

We proposed a large-scale, direct monocular SLAM system for omnidirectional cameras. Based on two different omnidirectional camera models, our system allows to use a wide range of classical dioptric or catadioptric imaging systems, including ones with a field of view exceeding $180^{\circ}$. The contribution of this paper is two-fold: (1) we explicitly formulate a camera model independent image registration algorithm for tracking and (2) derived a generic, accurate, and efficient way to perform stereo directly on the unified omnidirectional camera model, based on a parametric equation of the epipolar curves. We integrated these ideas into the LSD-SLAM framework and evaluated its real-time performance on a number of videos captured by a $185^{\circ}$ fisheye camera. We measure both an improvement of the accuracy of the localization and of its robustness to strong rotational movement compared to a standard camera. We also observe that even at relatively low resolutions $(240 \times 240)$, the localization accuracy surpasses the accuracy obtained when using a pinhole model, with a cropped field of view.

\section{REFERENCES}

[1] J. Engel, J. Sturm, and D. Cremers, "Camera-based navigation of a low-cost quadrocopter,' in IROS, 2012.

[2] M. Achtelik, M. Achtelik, S. Weiss, and R. Siegwart, "Onboard IMU and monocular vision based control for MAVs in unknown in- and outdoor environments," in ICRA, 2011.

[3] A. J. Davison, I. D. Reid, N. D. Molton, and O. Stasse, "Monoslam: Real-time single camera slam," IEEE Trans. Pattern Anal. Machine Intell., vol. 29, no. 6, pp. 1052-1067, 2007.

[4] M. Li and A. I. Mourikis, "High-precision, consistent EKF-based visual-inertial odometry," Int. J. Robot. Res., vol. 32, no. 6, pp. 690711, 2013

[5] A. Chiuso, P. Favaro, H. Jin, and S. Soatto, "Structure from motion causally integrated over time," PAMI, vol. 24, pp. 523-535, Apr 2002.

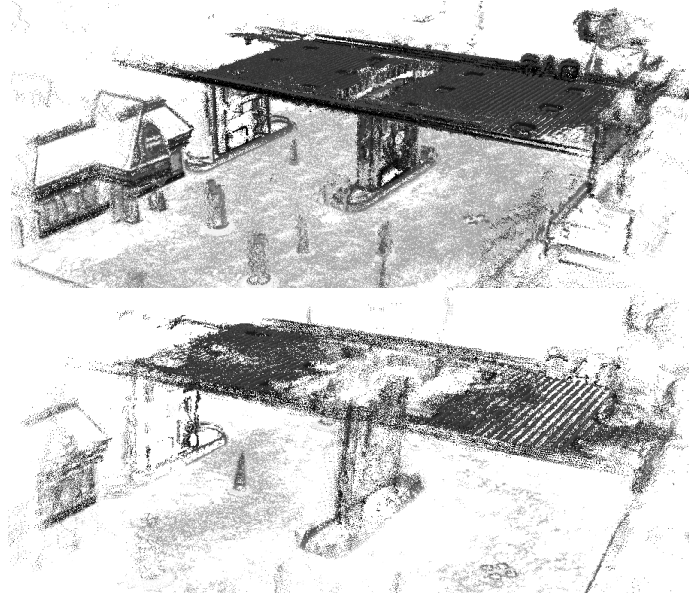

Fig. 11. Reconstruction evaluation. Final point cloud obtained on S1 trajectory for two different resolutions (top $480 \times 480$, bottom $240 \times 240$ ): The resolution has a very sensitive impact on the completeness and accuracy of the $3 \mathrm{D}$ reconstruction.

[6] G. Klein and D. Murray, "Parallel tracking and mapping for small ar workspaces," in ISMAR, 2007.

[7] S. Leutenegger, P. Furgale, V. Rabaud, M. Chli, K. Konolige, and R. Siegwart, "Keyframe-based visual-inertial slam using nonlinear optimization," in RSS, 2013.

[8] H. Strasdat, A. J. Davison, J. M. M. Montiel, and K. Konolige, "Double window optimisation for constant time visual SLAM," in ICCV, 2011.

[9] R. A. Newcombe, S. J. Lovegrove, and A. J. Davison, "DTAM: Dense tracking and mapping in real-time," in ICCV, 2011.

[10] J. Engel, T. Schoeps, and D. Cremers, "LSD-SLAM: Large-scale direct monocular SLAM," in ECCV, 2014.

[11] D. Scaramuzza and R. Siegwart, "Appearance-guided monocular omnidirectional visual odometry for outdoor ground vehicles," IEEE Trans. Robot., vol. 24, no. 5, pp. 1015-1026, 2008.

[12] J.-P. Tardif, Y. Pavlidis, and K. Daniilidis, "Monocular visual odometry in urban environments using an omnidirectional camera," in IROS, 2008.

[13] C. Silpa-Anan and R. Hartley, "Visual localization and loop-back detection with a high resolution omnidirectional camera," in Workshop on Omnidirectional Vision, 2005.

[14] D. Gutierrez, A. Rituerto, J. Montiel, and J. J. Guerrero, "Adapting a real-time monocular visual slam from conventional to omnidirectional cameras," in ICCV Workshops, 2011.

[15] A. Rituerto, L. Puig, and J. Guerrero, "Comparison of omnidirectional and conventional monocular systems for visual slam," 10th OMNIVIS with RSS, 2010

[16] M. Meilland, A. I. Comport, and P. Rives, "A spherical robot-centered representation for urban navigation," in IROS, 2010, pp. 5196-5201.

[17] D. Scaramuzza, A. Martinelli, and R. Siegwart, "A flexible technique for accurate omnidirectional camera calibration and structure from motion," in ICVS, 2006.

[18] C. Geyer and K. Daniilidis, "A unifying theory for central panoramic systems and practical implications," in ECCV, 2000.

[19] X. Ying and Z. Hu, "Can we consider central catadioptric cameras and fisheye cameras within a unified imaging model," in ECCV, 2004.

[20] J. P. Barreto, "Unifying image plane liftings for central catadioptric and dioptric cameras," in Imaging Beyond the Pinhole Camera. Springer, 2006, pp. 21-38.

[21] S. Baker and I. Matthews, "Lucas-kanade 20 years on: A unifying framework," IJCV, vol. 56, no. 3, pp. 221-255, 2004.

[22] J. Engel, J. Sturm, and D. Cremers, "Semi-dense visual odometry for a monocular camera," in ICCV, 2013.

[23] J. Maye, P. Furgale, and R. Siegwart, "Self-supervised calibration for robotic systems," in $I V$, June 2013.

[24] R. Mur-Artal, J. M. M. Montiel, and J. D. Tardós, "ORB-SLAM: a versatile and accurate monocular SLAM system," IEEE Trans. Robot. (accepted) arXiv preprint arXiv:1502.00956, 2015.

[25] J. Engel, J. Stueckler, and D. Cremers, "Large-scale direct slam with stereo cameras," in IROS, 2015. 